\title{
Spatially coupled LDPC codes for power line communications with impulsive noise
}

\author{
Inayat Ali, Hyunjae Lee, Yong Sung Kil, Ayaz Hussain, \\ and Sang-Hyo Kim ${ }^{\text {a) }}$ \\ Department of Electrical and Computer Engineering, \\ Sungkyunkwan University, Suwon 16419, Korea \\ a)iamshkim@skku.edu
}

\begin{abstract}
In this paper, we analyze and propose spatially coupled lowdensity parity-check (SC LDPC) codes for power line communications (PLC). With windowed-sequential encoding and decoding characteristics of SC LDPC codes, the low latency error correction with superior performance becomes the cornerstone for proposing these codes for PLC systems. The communication channels in PLC have impulsive noise, we use Bernoulli-Gaussian model which has heavy tail distribution for this channel. Through computer simulations, we compare these codes with block LDPC codes and show that SC LDPC codes perform better due to connectivity between coupled blocks.
\end{abstract}

Keywords: impulse noise channels, SC LDPC codes, power line communications, windowed decoder, Bernoulli-Gaussian model

Classification: Power devices and circuits

\section{References}

[1] C. Hsu, et al:: "Improving a power line communications standard with LDPC codes,” EURASIP J. Adv. Signal Process. 2007 (2007) 060839 (DOI: 10.1155/ 2007/60839).

[2] A. M. Tonello, et al.: "Power line communications: Understanding the channel for physical layer evolution based on filter bank modulation," IEICE Trans. Commun. E97.B (2014) 1494 (DOI: 10.1587/transcom.E97.B.1494).

[3] HomePlug: perfect fit for Smart Energy/Internet of Things (IoT) applications, http://www.homeplug.org/tech-resources/green-phy-iot/.

[4] J. Lin, et al.: "Impulsive noise mitigation in powerline communications using sparse bayesian learning," IEEE J. Sel. Areas Commun. 31 (2013) 1172 (DOI: 10.1109/JSAC.2013.130702).

[5] D. Middleton: "Procedures for determining the parameters of the first-order canonical models of class A and class B electromagnetic interference," IEEE Trans. Electromagn. Compat. EMC-21 (1979) 190 (DOI: 10.1109/TEMC. 1979.303731).

[6] T. Shongwey, et al:: "A study on impulse noise and its models," 18th IEEE International Symposium on Power Line Communications and its Applications (ISPLC) (2014) 12 (DOI: 10.1109/ISPLC.2014.6812360). 
[7] S. Kudekar, et al:: "Spatially coupled ensembles universally achieve capacity under belief propagation," IEEE Trans. Inf. Theory 59 (2013) 7761 (DOI: 10. 1109/TIT.2013.2280915).

[8] N. ul Hassan, et al.: "Improving code diversity on block-fading channels by spatial coupling," IEEE International Symposium on Information Theory (ISIT) (2014) 2311 (DOI: 10.1109/ISIT.2014.6875246).

[9] M. Shafieipour, et al.: "Robust turbo coded OFDM transceiver for power-line channels," IEICE Electron. Express 7 (2010) 1416 (DOI: 10.1587/elex.7. 1416).

[10] I. Ali, et al:: "Improving windowed decoding of SC LDPC codes by effective decoding termination, message reuse, and amplification," IEEE Access 6 (2017) 9336 (DOI: 10.1109/ACCESS.2017.2771375).

\section{Introduction}

With the recent rapid developments in the Internet of Things (IoT), PLC has become a viable source for communication between different IoT devices. PLC exploits the power line infrastructure existing in almost every home or office and utilizes the unused bandwidth in these power lines for data transmissions [1, 2]. HomePlug Green PHYтм uses IEEE 1901 standards for power line communication and is widely considered for IoT at home, indoor broadband and other home automation applications [3]. The communication systems in PLC usually experience impulsive noise due to the sharing of the same medium with heavy electrical appliances. For example, switching transients and switched-mode power supply can induce impulsive noise in the data stream [4]. The impulsive noise channel was first modeled by Middleton [5], known in many literatures as Middleton Class A noise. Another noise distribution similar to Middleton Class A is Bernoulli-Gaussian model, also known as Gaussian Mixture with Bernoulli distribution [6]. Due to its tractability, Bernoulli-Gaussian model is considered in modeling impulsive noise in this paper.

Error correction with SC LDPC codes has been shown to be capacity achieving due to threshold saturation property of these codes [7]. Multiple block LDPC codes are coupled together to form an SC LDPC code. The capacity achieving performance of these codes is due to falling dominoes-like decoding convergence starting from two terminated sides of their bipartite graph. In [8], it has been shown that the inter-block coupling characteristic of these codes increases the diversity of coding in non-ergodic channels. Therefore, in the impulse noise channel this characteristic is again exploited to get high coding gains. In [9], turbo codes were considered for error correction in PLC. Block LDPC codes were analyzed and proposed for PLC, more specifically HomePlug 1.0 in ROBO mode in [1]. In this paper, we propose and analyze SC LDPC codes for PLC and show that SC LDPC codes have more coding gain in impulsive noise channels.

\section{Impulsive noise channel model}

The Bernoulli-Gaussian model is a simple two-state impulse generating channel, in which the impulse state is a Bernoulli process with probability ' $p$ '. The sampled 


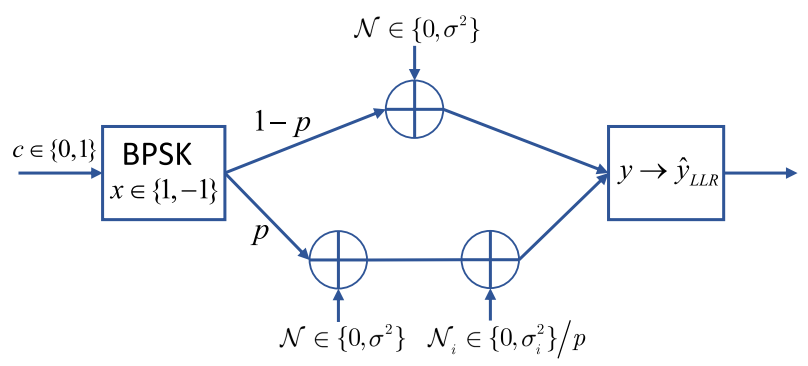

Fig. 1. Impulsive noise (Bernoulli-Gaussian) channel model

noise in this model is a mixture of background Gaussian noise and impulse noise that is also Gaussian distributed [6].

$$
\mathcal{X}_{\mathrm{BG}}\left(\sigma^{2}, k\right)=\mathcal{X}\left(\sigma^{2}, k\right)+\psi(k) \cdot \mathcal{X}_{i}\left(\sigma_{i}^{2}, k\right) / p,
$$

where $k \in \mathbb{Z}^{+}$is the index of bit sample, $\mathcal{X}\left(\sigma^{2}, k\right)$ is the Gaussian noise sample with variance $\sigma^{2}$ and probability density function (PDF) $\mathcal{N}\left(0, \sigma^{2}\right), \psi(k)$ is the impulse indicator variable defined by Bernoulli process:

$$
\psi(k)= \begin{cases}1, & \text { if } w(k) \leq p, \quad w(k) \sim \operatorname{Unif}(0,1) \\ 0, & \text { otherwise }\end{cases}
$$

Where $\operatorname{Unif}(0,1)$ is a uniform number generator in the range of 0 to 1 . The PDF of the Bernoulli-Gaussian impulsive noise channel is given by:

$$
F\left(\mathcal{X}_{\mathrm{BG}}\left(\sigma^{2}, k\right)\right)=(1-p) \cdot \mathcal{N}\left(0, \sigma^{2}\right)+p \cdot \mathcal{N}\left(0, \sigma^{2}+\sigma_{i}^{2}\right) .
$$

The PDF of this channel has a longer tail, thus they are sometimes called 'heavy tail distribution' channel. The variance of impulse noise $\sigma_{i}^{2}$ is characterized by the parameter $\Gamma=\sigma^{2} / \sigma_{i}^{2}$ (i.e the Gaussian to impulse noise power ratio). Fig. 1 pictorially describes the channel model defined above. The time-domain noise measurements of the impulsive noise channel are shown in Fig. 2. It can be noted that $p \in[0,1]$ defines the frequency of occurrence of impulse and the average amplitude of the impulse noise is determined by $\Gamma$.

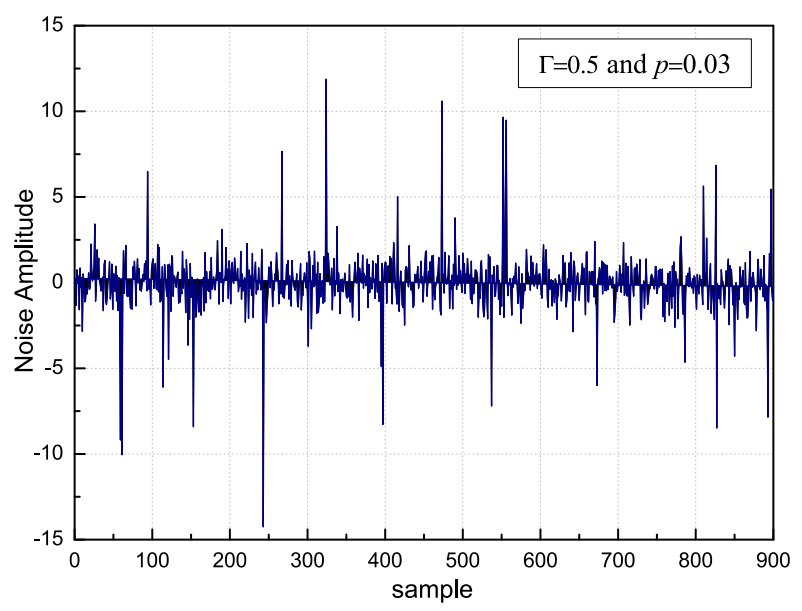

Fig. 2. Noise measurement. 


\section{Spatially coupled LDPC codes}

The convolutional LDPC codes, also known as the SC LDPC codes are shown to achieve capacity of general BMS channels universally (here, universal refers to the property that a single ensemble or code having similar behavior over other channels) [7]. In terms of protograph, these codes can be constructed by a coupling technique known as 'edge spreading' [10]. Coupling depth, also known as coupling width ' $w$ ' can be adjusted by applying various sequences of edge spreading that couple bit-nodes with check nodes $w$ spatial positions apart.

In practice, SC LDPC codes perform very well, but at the expense of some rate loss. Coupling of multiple LDPC blocks induces overhanging edges. These overhanging edges are connected with some extra check nodes, resulting in rate loss. However, if coupling length ' $L$ ' is taken large, this rate loss diminishes. In this paper, we follow the code construction method defined in [10] and only $(3,6) \mathrm{SC}$ LDPC code with $w=2$ is used.

\subsection{Windowed decoder}

In latency constrained applications similar to PLC, the BP decoding over the entire graph with large ' $L$ ' becomes infeasible. Due to diagonal band structure of the parity check matrix (PCM), the SC LDPC codes can be decoded sequentially with a windowed decoder. The decoding proceeds after receiving the bit-nodes inside the windowed decoder. The iterative BP decoder runs inside the window of size ' $W$ ' to decode the target symbols. The window slides down diagonally until all ' $L$ ' target symbols are decoded. Fig. 3 shows the PCM of a $(3,6)$ SC LDPC code and explains the dimension and the operation of the windowed decoder. Again, we follow the windowed decoding procedure defined in [10]. The complexity, memory, and latency of decoding is significantly reduced by localizing the BP decoder inside a window. When the statistics of the channel are known, the throughput of the decoder can be efficiently controlled by varying $W$.

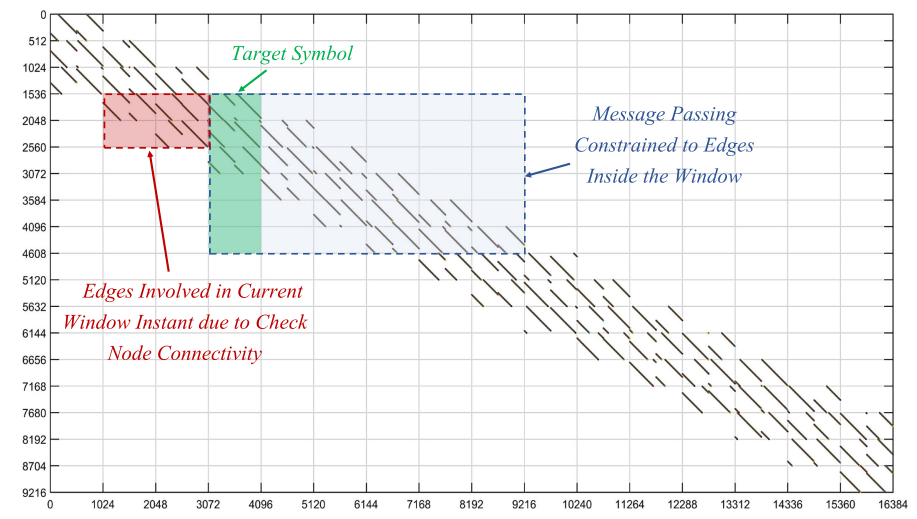

Fig. 3. PCM of $L=16(3,6)$ SC LDPC code and windowed decoding at 4 th decoding instant $(W=6)$.

\section{Numerical results}

To show the advantage of using SC LDPC codes over the PLC channel, computer simulations were conducted with different channel conditions (i.e $\Gamma$ and $p$ ). The 
length of the sub-block of SC LDPC code is kept equal to that of block LDPC code. Both codes were constructed from regular $(3,6)$ ensemble with quasi-cyclic permutation matrices of lifting factor $M=100$. The coupling length and width of the SC LDPC code were fixed as $L=100$ and $w=2$, respectively. The maximum number of decoding iterations is set as 100 .

\subsection{BP decoding performance}

Fig. 4 shows the BER performance of SC and block LDPC codes with different impulse channel parameter $\Gamma$ and $p$ (sub-block BER is considered for latter's case). In all channel conditions, the SC LDPC code outperforms the block LDPC code. For $\Gamma=0.1$ and $p=0.1$, the block LDPC code performance hits error-floor at approximately $10^{-3}$, whereas, SC LDPC code achieves BER of $10^{-6}$ without showing error floor.

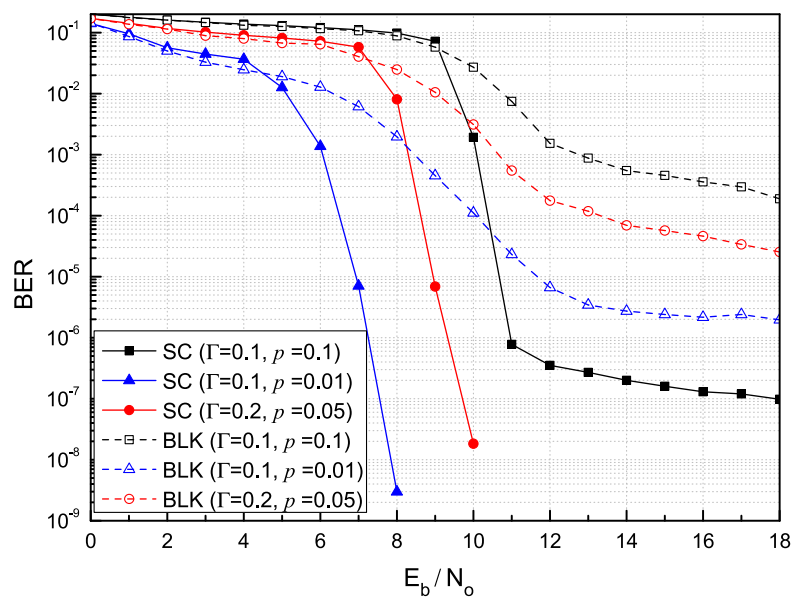

Fig. 4. BER performance comparison between SC and block LDPC codes over impulsive noise channel.

\subsection{Windowed decoding performance}

In latency constrained applications of PLC, WD can be used to provide robust error correction with low latency. For fair comparison, we compare the BER of block LDPC code with the sub-block BER of SC LDPC code decoded with WD (here, sub-block BER means the individual target symbol BER of WD). Fig. 5 shows the comparison between WD sub-block wise BER performance curves with the BER of block LDPC code. The SC LDPC code performs better than the block LDPC code, even with small $W=4$. The BER improves with the window size, therefore for moderate $W$, the SC LDPC codes exhibit close to the BP decoding performance. It was also observed that by increasing the maximum number of iterations the performance of WD improves remarkably. Whereas, this is not the case for block LDPC code. The coupling between consecutive blocks in the SC LDPC codes provides a better flow of information at bit-nodes. Hence, the high erroneous LLRs due to impulse noise need more decoding rounds to change their sign. 


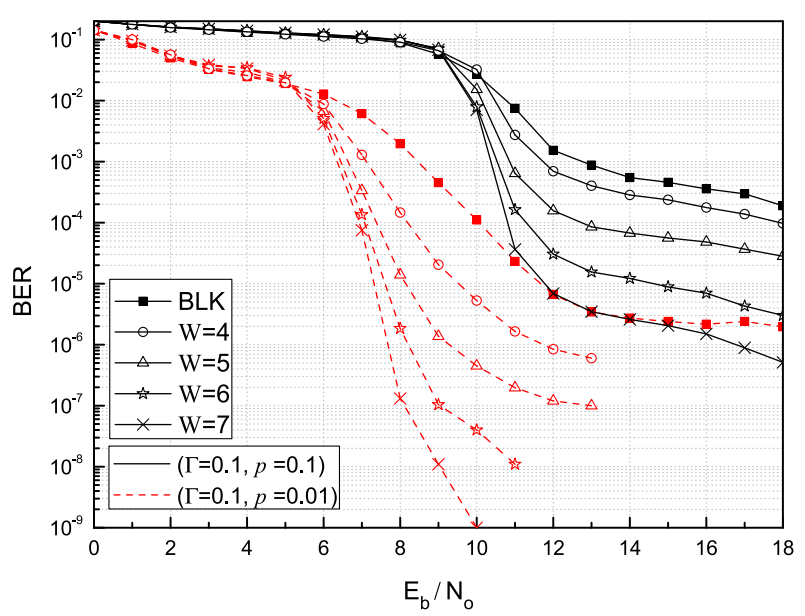

Fig. 5. BER performance comparison between SC LDPC code decoded with WD of different window sizes ' $W$ ' and block LDPC code.

\section{Conclusion}

In this paper, we analyzed SC LDPC codes over the power line communication medium, in which the channel is characterized by impulsive noise. We compared the error correcting performance of these codes with their block counterpart and showed that the SC LDPC codes have remarkably better performance. The SC LDPC codes can be sub-block wise sequentially decoded with low latency windowed decoder, therefore we also simulated and compared the windowed decoding performance of SC LDPC codes. Even with the small window size, the performance of SC LDPC codes is better than block LDPC codes. When the channel statistics are known, we can adjust the size of the windowed decoder for high throughput.

\section{Acknowledgments}

This research was supported by Basic Science Research Program through the National Research Foundation of Korea (NRF) funded by the Ministry of Science and ICT (NRF-2018R1A2B6004195) and (NRF-2015R1D1A1A01058975). 